\title{
Well Differentiated Liposarcoma
}

National Cancer Institute

\section{Source}

National Cancer Institute. Well Differentiated Liposarcoma. NCI Thesaurus. Code C4250.

A locally aggressive malignant neoplasm composed of mature adipocytes showing cell size variation and nuclear atypia. It is often associated with the presence of

hyperchromatic multinucleated stromal cells, and varying numbers of lipoblasts. There are three histologic subtypes, sclerosing, inflammatory, and spindle cell liposarcoma.

These tumors do not usually metastasize unless they undergo dedifferentiation. 